\title{
Choroidal structure determined by binarizing optical coherence tomography images in eyes with reticular pseudodrusen
}

This article was published in the following Dove Press journal:

Clinical Ophthalmology

27 April 2017

Number of times this article has been viewed

\section{Naonori Masuda \\ Masashi Kojima \\ Mariko Yamashita \\ Tomo Nishi \\ Nahoko Ogata}

Department of Ophthalmology, Nara

Medical University, Nara, Japan
Correspondence: Nahoko Ogata Department of Ophthalmology, Nara Medical University, 840 Shijo-cho, Kashihara, Nara, 634-8522, Japan Email ogata@naramed-u.ac.jp
Purpose: To compare the choroidal structure beneath the macular area in eyes with reticular pseudodrusen (RPD) and age-matched controls.

Methods: This study was performed at Nara Medical University Hospital, Japan. Twenty eyes of 14 patients ( $82.3 \pm 4.2$ years, mean \pm standard deviation) with RPD and 35 eyes of 20 agematched controls ( $81.5 \pm 6.0$ years) were studied. The choroidal structure was determined by binarizing the images obtained by enhanced depth imaging optical coherence tomography in all patients and controls. The total, luminal, and stromal choroidal areas were quantified by the binarization method.

Results: The total choroidal area of the eyes with RPD was significantly smaller than that of control eyes ( $P=0.001$, unpaired $t$-test). Both the luminal and stromal areas in eyes with RPD were significantly smaller than that of control eyes ( $P=0.001$, paired $t$-test), but there was no significant difference in the luminal/stromal ratio between eyes with RPD and control eyes.

Conclusion: The total, luminal, and stromal choroidal areas in eyes with RPD were smaller than those of the control eyes. The reduction of the choroidal luminal and stromal areas may be due to a loss of the oxygen demand of the choroid due to RPE dysfunction.

Keywords: reticular pseudodrusen, age-related macular degeneration, choroidal structure, binarization method

\section{Introduction}

Age-related macular degeneration (AMD) is the leading cause of blindness in older adults in industrialized countries. ${ }^{1}$ Reticular pseudodrusen (RPD) are seen ophthalmoscopically or in fundus photographs as small yellowish-white lesions arranged in a reticular pattern. ${ }^{2}$ They are important because they are high-risk signs for late-onset AMD. ${ }^{2-4}$ The pathophysiological mechanisms causing these lesions have not been conclusively determined. Several authors have reported that there is a significant correlation between the presence of RPD and the choroidal thickness. ${ }^{5-8}$ Querques et al demonstrated that the choroidal thickness and the volume of the choroid beneath the macular region in eyes with RPD were significantly decreased, ${ }^{6}$ but only a few studies have examined the individual choroidal structures, that is, the luminal and stromal structures, in eyes with RPD. This is probably because the luminal and stromal areas of the choroid are not morphologically arranged in a clear pattern.

Recently, Sonoda et al reported a new method to analyze the luminal and stromal areas of the choroid quantitatively. ${ }^{9,10}$ They used an open access software, ImageJ, to differentiate the luminal and stromal areas of the choroid and to quantify the size of 
these areas. ${ }^{9,10}$ To the best of the authors' knowledge, this has not been done on eyes with RPD.

Thus, the purpose of this study was to compare the individual choroidal structures of eyes with RPD to that of agematched controls. To accomplish this, images were obtained by enhanced depth imaging optical coherence tomography (EDI-OCT) from both types of eyes, and the images were binarized, quantified, and compared.

\section{Methods}

\section{Participants}

This was a cross-sectional observational study conducted at the Nara Medical University from April 2012 to August 2015. All examinations were performed between 11:00 and 15:00 hours to avoid diurnal variations of the choroidal structures. ${ }^{11,12}$ The protocol of this study conformed to the tenets of the Declaration of Helsinki, and it was approved by Internal Review Board of the Nara Medical University. An informed consent was obtained from all of the participants to perform the original measurements and to review their medical records.

Twenty eyes of 14 patients $(82.3 \pm 4.2$ years) with RPD were studied. In addition, 35 eyes of 20 age-matched healthy controls (81.5 \pm 6.0 years) were studied in the same way. RPD were considered to be present when small yellowishwhite lesions arranged in a reticular pattern were seen in the macular area. ${ }^{2}$ The visibility of the RPD was enhanced by infrared illumination.

All of the participants had a complete eye examination including measurements of the best-corrected visual acuity (BCVA) and intraocular pressure, slit-lamp biomicroscopy, ocular fundus examination, and spectral-domain optical coherence tomography (SD-OCT) recordings. Patients with neovascular AMD, chorioretinal atrophy in the macular area, myopia $>-3.0$ diopters (D), ${ }^{9}$ organic eye diseases, previous laser treatments, cataract surgery within 6 months, cataracts that made the fundus examination difficult, glaucoma, and any other retinal disorders were excluded. The visual acuity was measured with a standard Snellen chart, and the decimal visual acuity was converted to the logarithm of the minimal angle of resolution (logMAR) units for the statistical analyses.

\section{Optical coherence tomography (OCT)}

The choroidal area was measured on the images obtained by SD-OCT (Spectralis, Heidelberg Engineering, Heidelberg, Germany) with the EDI program from all patients and controls. All images were recorded by one of the authors or by an experienced ophthalmologist. Two observers who were masked as to whether the eye had RPD selected the best image and independently measured the choroidal areas. The final area was calculated as the arithmetic means of the two observers.

\section{Evaluation of choroidal area by binarizing EDI OCT images}

The binarization of the choroidal area was done with an open access software, ImageJ (version 1.50a; National Institutes of Health, Bethesda, MD, USA) according to the method described in detail by Sonoda et al. ${ }^{9}$ Briefly, the examined area was selected to be $1,500 \mu \mathrm{m}$ wide with margins $750 \mu \mathrm{m}$ nasal and $750 \mu \mathrm{m}$ temporal to the fovea. The choroidal area extended vertically from the RPE to the chorioscleral border, and the borders were set with the ImageJ ROI Manager. Three choroidal vessels with lumens $>100 \mu \mathrm{m}$ were randomly selected by the Oval Selection Tool on the ImageJ tool bar, and the average reflectivity of these areas was determined. The average brightness was set at the minimum value to minimize the noise in the OCT image. Then, the image was converted to 8 bits and adjusted by the Niblack Auto Local Threshold. The binarized image was converted to red, green, and blue images again, and the luminal area was determined by using the Threshold Tool. After adding the distance between the pixels, the choroidal area, luminal area, and stromal area were automatically calculated. The light pixels were defined as the stromal area, and the dark pixels were defined as the luminal area.

\section{Statistical analyses}

All statistical analyses were performed with a commercial analytical package (SPSS Statistics 19 for Windows; IBM Corporation, Armonk, NY, USA). The data are expressed as the means \pm standard deviations (SDs). The interobserver reproducibility was evaluated by intraclass correlation coefficients (ICCs).

The areas of the subfoveal choroid including the stromal area and luminal area in eyes with RPD were compared with that of the controls by unpaired $t$-tests. A $P$-value $<0.05$ was considered to be statistically significant.

\section{Results}

The demographic characteristics of the RPD group and the control group are shown in Table 1. The difference in the age between the RPD group ( $82.3 \pm 4.2$ years) and the control group ( $81.5 \pm 6.0$ years) was not significant $(P>0.05)$. The mean BCVA was $0.11 \log$ MAR units in the eyes with RPD which was significantly better than the BCVA in the control eyes at $0.23 \log$ MAR units $(P<0.05)$. 
Table I Characteristics of eyes with RPD and those of the control

\begin{tabular}{llll}
\hline Characteristics & $\begin{array}{l}\text { Eyes with } \\
\text { RPD }(\mathbf{n}=\mathbf{2 0})\end{array}$ & $\begin{array}{l}\text { Controls } \\
(\mathbf{n}=\mathbf{3 5})\end{array}$ & P-value $^{\mathbf{a}}$ \\
\hline Mean age (years) & $82.3 \pm 4.2$ & $81.5 \pm 6.0$ & 0.648 \\
Mean IOP (mmHg) & $12.8 \pm 1.8$ & $14.1 \pm 2.8$ & 0.061 \\
Refractive error, & $-0.20 \pm 1.54$ & $-0.24 \pm 1.62$ & 0.923 \\
spherical equivalent (D) & & & \\
BCVA (logMAR) & $0.11 \pm 0.18$ & $0.23 \pm 0.13$ & $<0.05$ \\
\hline
\end{tabular}

Notes: All values are expressed as mean \pm standard deviation (range). ${ }^{a}$ Unpaired t-test.

Abbreviations: RPD, reticular pseudodrusen; IOP, intraocular pressure; BCVA, best-corrected visual acuity; logMAR, logarithm of the minimal angle of resolution.

All health controls and phackic eyes and three of RPD patients had peudophakic eyes. The mean total choroidal area, luminal area, and stromal area in eyes with RPD were significantly smaller than the corresponding structures of the control eyes $\left(193,370.4 \pm 53,653.0 \mu \mathrm{m}^{2}\right.$ vs $273,915.6 \pm 78,764.0 \mu \mathrm{m}^{2}$, $127,505.7 \pm 78,764.4 \mu \mathrm{m}^{2}$ vs $183,152.2 \pm 55,666.4 \mu \mathrm{m}^{2}$,

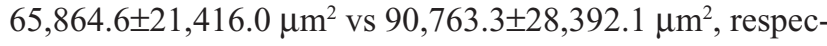
tively, $P=0.001, P=0.001, P=0.003$; unpaired $t$-test, respectively). However, the mean ratio of the luminal area to the choroidal $(0.66 \pm 0.05)$ in the eyes with RPD was not significantly different from that in the eyes of controls $(0.66 \pm 0.05$; $P=0.6$, Table 2, Figure 1).

The interobserver reproducibility was very high with ICC $=0.83$ for the total choroidal area and 0.83 for the stromal area. It was also high for the luminal area with ICC $=0.78$.

\section{Representative RPD eye and control eye}

EDI-OCT images and the converted binary images of a representative RPD eye and a control eye are shown in Figure 2. The luminal areas of the choroid are displayed as black areas and stromal areas are displayed as white areas. In the RPD patient, the EDI-OCT image shows that the hyperreflective areas are located above the retinal pigment

Table 2 Results of choroidal binarization to quantify the total, luminal, and stromal choroidal areas in patients with RPD and in control subjects

\begin{tabular}{llll}
\hline & $\begin{array}{l}\text { Eyes with } \\
\text { RPD }(\mathbf{n}=\mathbf{2 0})\end{array}$ & $\begin{array}{l}\text { Controls } \\
(\mathbf{n}=\mathbf{3 5})\end{array}$ & $\boldsymbol{P}_{\text {-value }}{ }^{\mathbf{a}}$ \\
\hline Total choroidal & $193,370.4 \pm 53,653.0$ & $273,915.6 \pm 78,764.4$ & $0.00 \mathrm{I}$ \\
area $\left(\mu \mathrm{m}^{2}\right)$ & & & \\
Luminal area $\left(\mu \mathrm{m}^{2}\right)$ & $127,505.7 \pm 35,612.8$ & $183,152.2 \pm 55,666.4$ & $0.00 \mathrm{I}$ \\
Stromal area $\left(\mu \mathrm{m}^{2}\right)$ & $65,864.6 \pm 21,416.0$ & $90,763.3 \pm 28,392.0$ & 0.003 \\
Luminal ratio & $0.66 \pm 0.05$ & $0.66 \pm 0.05$ & 0.6 \\
\hline
\end{tabular}

Notes: All values are expressed as mean \pm standard deviation (range). ${ }^{\text {anpaired }}$ t-test.

Abbreviation: RPD, reticular pseudodrusen.

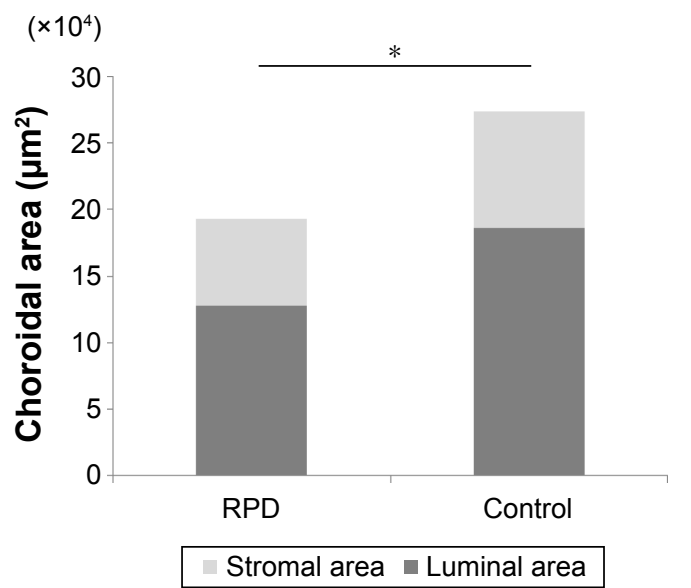

Figure I Subfoveal choroidal areas of EDI-OCT image in eyes with RPD and control eyes.

Notes: The choroidal, luminal, and stromal areas in eyes with RPD were significantly smaller than those of the control eyes ( $* P=0.00$ I, unpaired $t$-test).

Abbreviations: EDI-OCT, enhanced depth imaging optical coherence tomography; RPD, reticular pseudodrusen.

epithelium. The choroidal and luminal area of an eye with RPD are smaller than those of a control eye.

\section{Discussion}

The results showed that the mean total, luminal, and stromal choroidal areas of eyes with RPD were significantly smaller than those of control eyes. These findings confirm the earlier reports that the choroid was significantly thinner in eyes with RPD. ${ }^{5-8}$ The difference in the ratio of the luminal area to the total choroidal area of eyes with RPD was not significantly different from the ratio of the control eyes. The healthy controls were mainly hospitalized for cataract surgery. Thus, they had denser cataracts than patients who had RPD, although their cataracts did not disturb the OCT examination; therefore, they might have poorer BCVA than that of RPD group.

The pathophysiological mechanisms underlying the formation of the RPD lesions have not been conclusively determined. Querques et al also found that the choroid was thinner in eyes with RPD than in eyes with early AMD. They postulated that the pseudodrusen were associated with choroidal fibrosis which led to the choroidal thinning. ${ }^{13}$ Later, Sarks et al evaluated eyes with RPD and reported that pseudodrusen were due to the accumulation of subretinal materials. ${ }^{14}$ Spaide et al hypothesized that RPD were mainly caused by a dysfunction of the retinal pigment epithelium (RPE). ${ }^{15}$ They reported that older, highly myopic patients with choroidal thinning did not have an increased incidence of pseudodrusen, and they suggested that the choroidal thinning or decreased choroidal blood flow in older patients was not the cause of 

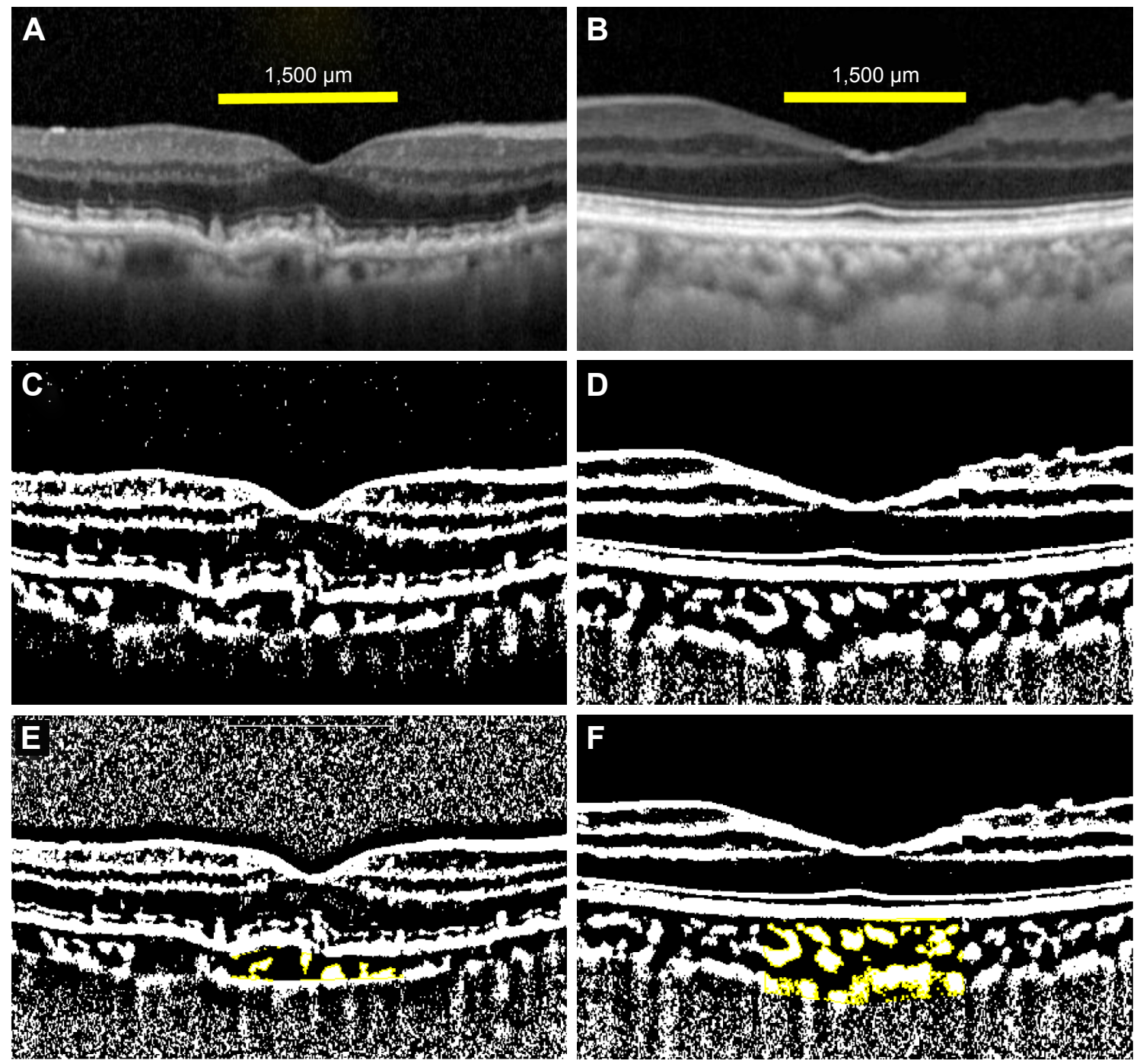

Figure 2 EDI-OCT images of an eye with RPD and a control eye.

Notes: The EDI-OCT image of the eye with RPD (A) was converted to a binary image (C) by using the Imagej software. An EDI-OCT image of the control eye (B) was converted to a binary image (D). The choroidal area measured was I,500 $\mu \mathrm{m}$ wide with the margins $750 \mu \mathrm{m}$ nasal and $750 \mu \mathrm{m}$ temporal to the fovea. Vertically, the area extended from the retinal pigment epithelium to the chorioscleral border. The yellow area is the measured area of the choroid (E and $\mathbf{F})$.

Abbreviations: EDI-OCT, enhanced depth imaging optical coherence tomography; RPD, reticular pseudodrusen.

pseudodrusen. ${ }^{16}$ Instead, they suggested that a reduction in RPE function may lead to a dysfunction in the transport mechanism between the RPE and the Müller cells, resulting in an accumulation of the materials such as unesterified cholesterol, apolipoprotein E, complement, and vitronectin in the subretinal space. ${ }^{17}$ Such materials would then block the transport of the shed outer segments to the RPE, resulting in further accumulation of materials in the subretinal space. The accumulation can then lead to an attenuation of the photoreceptor metabolic activity, which they suggested might explain the choroidal thinning in eyes with RPD. ${ }^{15}$ However, this suggestion has not been proven. McLeod et al studied postmortem choroids and demonstrated that RPE atrophy occurred first followed by choricapillaris degeneration in eyes with geographic atrophy. ${ }^{18}$ They postulated that the shrinkage of choroidal vessels might be caused by lack of vascular endothelial growth factor (VEGF) secreted by RPE cells. As geographic atrophy is related to RPD,${ }^{19}$ it is possible that a similar event may occur in eyes with RPD.

Ueda-Arakawa et al studied the choroidal thickness in eyes with RPD, and they demonstrated thinner choroidal vessels in en face images obtained by high penetration swept source OCT..$^{20}$ The results of the present study are consistent with theirs. Corvi et al reported a progressive thinning of the total choroidal area involving both the luminal and stromal areas in patients with RPD. ${ }^{21}$ They did not find a change in the ratios of the luminal-stromal area to the total choroidal area. The findings of this study are similar to their results, although they reported that the mean ratio of the luminal to the total choroidal area in eyes with RPD was smaller than 
that of control eyes. Patients who were older than their cohort were studied, and the stromal area became thinner with the reduction of the luminal area with aging. ${ }^{22-24}$

The reduction of the choroidal luminal and stromal areas may be due to a loss of the oxygen demand of the choroid or lack of VEGF secretion due to RPE dysfunction. The results of the luminal area in eyes of the control group were smaller than that reported by Sonoda et al, ${ }^{9}$ which may be because of the older participants in this cohort.

There are some limitations in this study. This was a retrospective study, and the selection of the cohorts was not entirely free of selection bias. In addition, the findings were made on a small number of Japanese subjects. Further studies with a larger number of subjects will be necessary.

\section{Conclusion}

This study showed that the total, luminal, and stromal choroidal areas in eyes with RPD were significantly smaller than those of control eyes. The findings of the study should help determine the pathogenesis of RPD.

\section{Disclosure}

The authors report no conflicts of interest in this work.

\section{References}

1. Lim LS, Mitchell P, Seddon JM, Holz FG, Wong TY. Age-related macular degeneration. Lancet. 2012;379:1728-1738.

2. Zweifel SA, Imamura Y, Spaide TC, Fujiwara T, Spaide RF. Prevalence and significance of subretinal drusenoid deposits (reticular pseudodrusen) in age-related macular degeneration. Ophthalmology. 2010;117(9):1775-1781.

3. Smith RT, Sohrab MA, Busuioc M, Barile G. Reticular macular disease. Am J Ophthalmol. 2009;148(5):733-743.

4. Schmitz-Valckenberg S, Steinberg JS, Fleckenstein M, Visvalingam S, Brinkmann CK, Holz FG. Combined confocal imaging laser ophthalmoscopy and spectral-domain optical coherence tomography imaging of reticular drusen associated with age-related macular degeneration. Ophthalmology. 2010;117(6):1169-1176.

5. Alten F, Clemens CR, Heiduschka P, Eter N. Localized reticular pseudodrusen and their topographic relation to choroidal watershed zones and changes in choroidal volumes. Invest Ophthalmol Vis Sci. 2013;54(5):3250-3257.

6. Querques G, Querques L, Forte R, Massamba N, Coscas F, Souied EH. Choroidal changes associated with reticular pseudodrusen. Invest Ophthalmol Vis Sci. 2012;53(3):1258-1263.

7. Garg A, Oll M, Yzer S, et al. Reticular pseudodrusen in early age-related macular degeneration is associated with choroidal thinning. Invest Ophthalmol Vis Sci. 2013;54(10):7075-7081.

Clinical Ophthalmology

\section{Publish your work in this journal}

Clinical Ophthalmology is an international, peer-reviewed journal covering all subspecialties within ophthalmology. Key topics include: Optometry; Visual science; Pharmacology and drug therapy in eye diseases; Basic Sciences; Primary and Secondary eye care; Patient Safety and Quality of Care Improvements. This journal is indexed on Submit your manuscript here: http://www.dovepress.com/clinical-ophthalmology-journal
8. Switzer DW Jr, Mendonça LS, Saito M, Zweifel SA, Spaide RF. Segregation of ophthalmoscopic characteristics according to choroidal thickness in patients with early age-related macular degeneration. Retina. 2012;32(7):1265-1271.

9. Sonoda S, Sakamoto T, Yamashita T, et al. Choroidal structure in normal eyes and after photodynamic therapy determined by binarization of optical coherence tomographic images. Invest Ophthalmol Vis Sci. 2014;55(6):3893-3899.

10. Sonoda S, Sakamoto T, Yamashita T, et al. Luminal and stromal areas of choroid determined by binarization method of optical coherence tomographic images. Am J Ophthalmol. 2015;159(6):1123-1131.e1.

11. Brown JS, Flitcroft D, Ying GS, Francis EL, et al. In vivo human choroidal thickness measurements: evidence for diurnal fluctuations. Invest Ophthalmol Vis Sci. 2009;50(1):5-12.

12. Tan CS, Ouyang Y, Ruiz H, Sadda SR. Diurnal variation of choroidal thickness in normal, healthy subjects measured by spectral domain optical coherence tomography. Invest Ophthalmol Vis Sci. 2012;53(1): 261-266.

13. Arnold JJ, Sarks SH, Killingsworth MC, Sarks JP. Reticular pseudodrusen. A risk factor in age-related maculopathy. Retina. 1995;15(3): 183-191.

14. Sarks J, Arnold J, Ho IV, Sarks S, Killingsworth M. Evolution of reticular pseudodrusen. Br J Ophthalmol. 2011;95(7):979-985.

15. Mrejen S, Spaide RF. The relationship between pseudodrusen and choroidal thickness. Retina. 2014;34(8):1560-1566.

16. Spaide RF. Outer retinal atrophy after regression of subretinal drusenoid deposits as a newly recognized form of late age-related macular degeneration. Retina. 2013;33(9):1800-1808.

17. Rudolf M, Malek G, Messinger JD, Clark ME, Wang L, Curcio CA. Sub-retinal drusenoid deposits in human retina: organization and composition. Exp Eye Res. 2008;87(5):402-408.

18. McLeod DS1 Grebe R, Bhutto I, Merges C, Baba T, Lutty GA. Relationship between RPE and choriocapillaris in age-related macular degeneration. Invest Ophthalmol Vis Sci. 2009;50(10):4982-4991.

19. Marsiglia M, Boddu S, Bearelly S, et al. Association between geographic atrophy progression and reticular pseudodrusen in eyes with dry age-related macular degeneration. Invest Ophthalmol Vis Sci: 2013; 54(12):7362-7369.

20. Ueda-Arakawa N, Ooto S, Ellabban AA, et al. Macular choroidal thickness and volume of eyes with reticular pseudodrusen using swept-source optical coherence tomography. Am J Ophthalmol. 2016; 157(5):994-1004.

21. Corvi F, Souied EH, Capuano V, Costanzo E, et al. Choroidal structure in eyes with drusen and reticular pseudodrusen determined by binarisation of optical coherence tomographic images. Br J Ophthalmol. 2016; pii: bjophthalmol-2016-308548.

22. Ikuno Y, Kawaguchi K, Nouchi T, Yasuno Y. Choroidal thickness in healthy Japanese subjects. Invest Ophthalmol Vis Sci. 2010;51(4): 2173-2176.

23. Ding X, Li J, Zeng J, et al. Choroidal thickness in healthy Chinese subjects. Invest Ophthalmol Vis Sci. 2011;52(13):9555-9560.

24. Ruiz-Moreno JM, Flores-Moreno I, Lugo F, Ruiz-Medrano J, Montero JA, Akiba M. Macular choroidal thickness in normal pediatric population measured by swept-source optical coherence tomography. Invest Ophthalmol Vis Sci. 2013;54(1):353-359.

\section{Dovepress}

PubMed Central and CAS, and is the official journal of The Society of Clinical Ophthalmology (SCO). The manuscript management system is completely online and includes a very quick and fair peer-review system, which is all easy to use. Visit http://www.dovepress.com/ testimonials.php to read real quotes from published authors. 